\title{
HyGen: generating random graphs with hyperbolic communities
}

\author{
Saskia Metzler ${ }^{1 *}$ and Pauli Miettinen ${ }^{2}$
}

${ }^{*}$ Correspondence:

saskia.metzler@mpi-inf.mpg.de

${ }^{1}$ Max-Planck-Institute for

Informatics, Saarland Informatics Campus, 66123, Saarbrücken,

Germany

Full list of author information is

available at the end of the article

\begin{abstract}
Random graph generators are necessary tools for many network science applications. For example, the evaluation of graph analysis algorithms requires methods for generating realistic synthetic graphs. Typically random graph generators are generating graphs that satisfy certain global criteria, such as degree distribution or diameter. If the generated graph is to be used to evaluate community detection and mining algorithms, however, the generator must produce realistic community structure, as well. Recent research has shown that a clique is not necessarily a realistic community structure, necessitating the development of new graph generators. We propose HYGEN, a random graph generator that leverages the recent research on non-clique-like communities to produce realistic random graphs with hyperbolic community structure, degree distribution, and clustering coefficient. Our generator can also be used to accurately model time-evolving communities.
\end{abstract}

Keywords: Random graph generators, Community detection, Hyperbolic community structure

\section{Introduction}

Modular structure is a characteristic of real-world networks. Its constituents, or communities, typically display specific patterns of connectivity. Especially in social networks, communities show a pronounced core-tail structure: a small fraction of the members have strong ties to each other and form the core. The majority of members only have ties to the core and not to each other (Laumann and Pappi 1976; Alba and Moore 1978; Morgan et al. 1997; Reed and Selbee 2001; Panzarasa et al. 2009; Metzler et al. 2019). This kind of intra-community structure is well described by a hyperbolic model (Araujo et al. 2014; Metzler et al. 2016). The hyperbolic model can express the particular core-tail structure which is frequently observed in real-world networks. It also encompasses power law-like connectivity and is suitably general to represent clique-like as well as star-like patterns of connectivity (see Fig. 1b).

Understanding network organization is a primary goal of social sciences. Reaching this goal requires not only adequate models, but also suitable community detection algorithms. The quality of community detection algorithms is best tested on real-world data. This, however, requires significant amounts of testing data with reliable labels. Manual labelling is qualitatively the most adequate. In contrast, any automated community labelling procedure implies a comparison of the community detection algorithm under test to another procedure of detecting the communities. A favourable alternative to obtain

(c) The Author(s). 2019 Open Access This article is distributed under the terms of the Creative Commons Attribution 4.0 International License (http://creativecommons.org/licenses/by/4.0/), which permits unrestricted use, distribution, and reproduction in any medium, provided you give appropriate credit to the original author(s) and the source, provide a link to the Creative Commons license, and indicate if changes were made. 


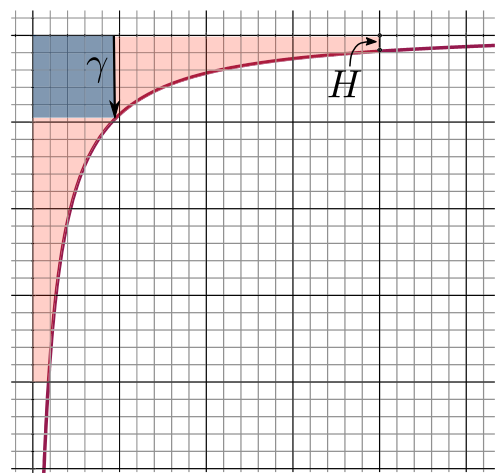

(a)
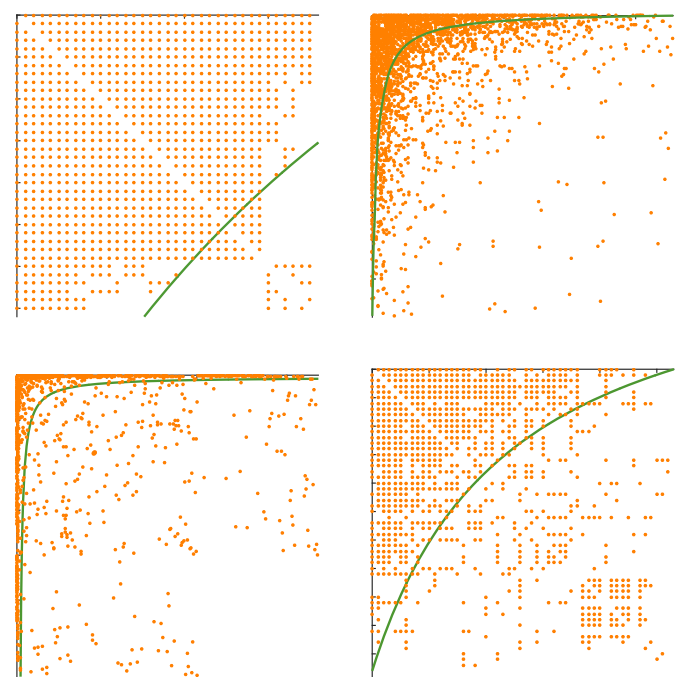

(b)

Fig. 1 Visualization of parameters for the hyperbolic model, and real-world examples. Models are shown on the degree-ordered adjacency matrix of each community. Customarily, the positive domain of the coordinate system is plotted from top left. Orange dots represent edges. The solid line visualizes the hyperbolic model. $\mathbf{a} \gamma$ and $H \mathbf{b}$ Examples of hyperbolic communities from (Metzler et al. 2016)

large amounts of reliably labelled testing data is to use random graph generators that create graphs with similar properties as real-world templates.

Most random graph generators aim at modelling global properties of the graphs, such as degree distribution, clustering coefficient, or effective diameter. Hence, models such as Erdős and Rényi (1959), Albert and Barabási (2002), or the Forest fire model (Leskovec et al. 2005) do not generate community structure and are not applicable to community detection. Similarly, graph expansion (Park and Kim 2018; Zhang and Tay 2016) and hyperbolic geometry (Krioukov et al. 2010) models focus on the global modelling of real-world graphs. Kronecker graphs (Leskovec et al. 2010) can be interpreted to have a community structure, but due to the recursive structure, the constructed communities have symmetries in size and shape not observed in real-world graphs.

Specialised random graph generators are proposed to generate graphs with a community structure. The best-known among them is the stochastic block model (SBM) (Holland et al. 1983), which is probably the most popular model to study community detection and clustering techniques (see Abbe (2017) for a detailed discussion). Despite its popularity, SBM has severe limitations, as it cannot incorporate variable inter-community degree distributions. To address this, degree-corrected SBMs (DC-SBM) (Karrer and Newman 2011) incorporate an additional degree parameter for each vertex. This allows modelling uneven edge probabilities. As pointed out by Zhu et al. (2014), DC-SBMs use the degrees of vertices as parameters, implying that the model cannot separate vertices based on degree even when that would be the correct partitioning. This limitation is overcome by degree-generated SBMs (Zhu et al. 2014). Degree-generated SBMs treat the expected degree of each vertex as generated from prior distributions, such as power laws, whose exponents vary from one community to another. Our model further differs from these, as we assume that intra- and inter-community edges have different probabilities. 
The R-MAT generator (Chakrabarti et al. 2004) is another popular model for generating random graphs with community structure. It is based on recursively subdividing the adjacency matrix into four equally sized partitions and distributing the edges within the partitions according to partition-specific probabilities. This approach allows it to mimic the degree distributions of real-world graphs, but restricts the shape of the constructed communities.

A third popular model for testing community detection algorithms is the LancichinettiFortunato-Radicchi (LFR) benchmark (Lancichinetti et al. 2008), which extends the Girvan-Newman benchmark (Girvan and Newman 2002). Unlike SBM or R-MAT, LFR can produce overlapping community structures and weighted and directed graphs. Compared to Girvan-Newman, LFR emphasizes the heterogeneous distributions of node degrees and community size. Yet, LFR generates near-uniform intra-community degree distributions, violating the assumption of non-clique-like community structures.

Orman et al. (2013) examine variations of LFR which achieve more realism with respect to transitivity and degree correlation in the generated graphs by choosing alternate random models for the initial step of the algorithm. In recent work, Fagnan et al. (2018) propose a generalization of LFR which follows the evolution patterns and characteristics of real networks. Unlike the present work, these works concentrate on overlapping communities.

To the best of our knowledge, however, no existing random graph generator is designed to model graphs consisting of hyperbolic communities. As many real-world graphs seem to follow a hyperbolic or core-tail model, we introduce a novel random graph generator to fill this gap. HYGEN generates modular networks with realistic intra-community structures using parameter distributions derived from observations on real graphs.

\section{Contributions}

This article extends our previous work on HyGEN (Metzler and Miettinen 2019). We present the model in more detail and have improved the exposition and analysis thereof. Our novel contributions in this work are the following:

- We present an alternative formulation of the HYGEN model as a graphon.

- We show that the graphon model is particularly suitable for modelling time-evolving communities.

- We show empirically that existing random graph generators are not suitable for generating hyperbolic community structure.

\section{The HyGen Model}

As discussed above, and as we will show later, the aforementioned random graph models do not generate communities that would have the kind of core-tail structure that is commonly observed in real-world social networks (see, e.g Araujo et al. (2014), Metzler et al. (2016, 2019)). To address that, we propose the HYGEN random graph generator that is based on the hyperbolic community model of Metzler et al. (2016). For the sake of completeness, we will first present the model of Metzler et al. (2016) before presenting the HYGEN model for one and multiple communities. Finally, we provide a different sampling approach, based on graphons, that is particularly suitable for time-evolving communities. Table 1 contains a list of symbols used throughout this article. 


\section{The hyperbolic community model}

The hyperbolic community model of Metzler et al. (2016) generates undirected graphs. Let $G=(V, E)$ be an undirected graph with $|V|=n$ and $|E|=m$. Assume that the vertices are assigned a unique number from $\{0, \ldots, n-1\}$ in an arbitrary manner. We use pair $(u, v)$ to denote both a pair of vertices and the (potential) undirected edge between them.

A community of $G$ is a tuple $\left(V_{C}, \pi_{C}, \Theta_{C}\right)$ where $V_{C} \subseteq V$ are the nodes of the community with $n_{C}=\left|V_{C}\right|, \pi_{C}: V_{C} \rightarrow\left\{0, \ldots, n_{C}-1\right\}$ is a permutation of the nodes that maps the nodes of a community to a set of indices $\left\{0, \ldots, n_{C}-1\right\}$, and $\Theta_{C}$ is a set of parameters that defines the shape of the community. We assume that the permutation $\pi_{C}$ orders the nodes in descending order according to their intra-community degree $\operatorname{deg}_{C}(v)=\left|\left\{u \in V_{C}:(v, u) \in E\right\}\right|$.

The key feature of the hyperbolic community model is that not all edges between nodes in $V_{C}$ are necessarily part of the community $V_{C}$. Which edges are part of the community is defined using a function $f:\left\{0, \ldots, n_{C}-1\right\} \times\left\{0, \ldots, n_{C}-1\right\} \times \Theta_{C} \rightarrow\{0,1\}$, the model of the community. The function $f$ takes a pair of permuted node indices and the set of parameters for the community, and decides whether the edge between the nodes is part of the community. An edge $(u, v)$ is a part of the community $C$ if

$1 \quad u \in V_{C}$ and $v \in V_{C}$; and

$2 f\left(\pi_{C}(u), \pi_{C}(v), \Theta_{C}\right)=1$.

In what follows we will mostly omit writing the permutation. Instead, when it is clear from the context, we will denote vertices by their indices in the permutation; for instance, $v, u$, and $w$ could be denoted by $i, j$, and $h$, with the meaning that $i=\pi_{C}(v), j=\pi_{C}(u)$, and $h=\pi_{C}(w)$.

Metzler et al. (2016) study three different models, called hyperbolic, fixed, and mixture. Despite being seemingly different, the three models are equivalent (Metzler et al. 2016), and we will use them interchangeably. For the sake of completeness, we will present two of them, hyperbolic and fixed, here, as they are the two models we will need the most. The hyperbolic model takes two parameters, $p$ and $\theta$, and the model function $f_{\text {hyperbolic }}$ is

$$
f_{\text {hyperbolic }}(i, j, p, \theta)=[(i+p)(j+p) \leq \theta],
$$

where we used the Iverson bracket notation: $[P]=1$ if proposition $P$ is true and $[P]=0$ otherwise. The hyperbolic model makes the hyperbola shape of the community obvious, but otherwise the parameters are not straight forward to interpret. The $f$ ixed model has more interpretable parameters: $\gamma$ defines the size of the core of the community and $H$ indicates how thick the tail is. A core of the community is a subcommunity of people that are known by (almost) everybody else in the community, while the tail consists of the rest of the members in the community (see Fig. 1a) ${ }^{1}$.

Metzler et al. (2016) show that we can transform the parameters of $f$ ixed to the parameters of hyperbolic using the following equations (Metzler et al. (2016), Eqs. (7) and (8)):

\footnotetext{
${ }^{1}$ The terms core and periphery are also used for similar structures (Borgatti and Everett 1999). We use the notion of core and tail instead since the hyperbolic model allows for more shape variations than the term periphery implies: tails may get progressively thinner while nodes in the periphery are assumed to be evenly connected to the core.
} 


$$
\begin{aligned}
& p=\frac{\gamma^{2}-\left(n_{C}-1\right) H}{\left(n_{C}-1\right)+H-2 \gamma} \\
& \theta=\frac{(\gamma-H)^{2}\left(\gamma-n_{C}-1\right)^{2}}{\left(n_{C}-1+H-2 \gamma\right)^{2}} .
\end{aligned}
$$

\section{Single community}

The model for a single community is straight forward and we express it via the fixed model. Assume, for now, that the size of the community, $n_{C}$, is given. To define the community in $\mathrm{f} i \mathrm{xed}$, we need to have the two parameters, $\gamma$ and $H$. In our model, we assume that the relative $\gamma, \gamma_{R}=\gamma / n_{C}$, follows the normal distribution with some mean $\mu$ and variance $\sigma^{2}$. Assuming we have sampled some $\gamma=n_{C} \gamma_{R}$, we will sample $H$ by assuming that $H / \gamma \sim \operatorname{Exponential}(\lambda)$ (we will motivate these distributions later using experiments with real-world data sets).

After we have sampled $\gamma$ and $H$, we can generate a perfect community $C$ using the fixed $\gamma, H$ model. The community $C$ will have no noise, that is, no edges between the nodes in the tail of the community and no missing edges within the community. As this is an unrealistic assumption, we will apply uniform noise to remove edges from the community and add edges between tail nodes. For this, we will need two more parameters, $d_{\text {in }}$ and $d_{\text {out }}$, that describe the densities of the community and of the area outside the community; a fraction of $1-d_{\text {in }}$ edges within the community are removed and a fraction of $d_{\text {out }}$ edges outside the community (i.e. between tail nodes) is added.

\section{Multiple communities}

To generate multiple communities, we use an approach similar to SBMs, and generate individual communities independently of each other. For each community, we will draw the size $n_{C}$ from distribution $D_{\text {size }}$. Our experiments suggest using the generalized extreme value distribution as the distribution of the sizes, but the power law distribution, as used by Lancichinetti et al. (2008), is a viable alternative.

After the community size is sampled, we can sample $\gamma$ and $H$ as explained above. Do note that the way we sample them is independent of the community size, and hence we can use the same distributions for every community.

After we have sampled a noise-free community, we will remove some of its edges to obtain the desired "inside" density $d_{\text {in }}$ and plant the community to the graph. The planting happens so that the communities do not overlap, similar to SBMs, but we can permute the order of the nodes, so that the hyperbolic shape is not immediately obvious. After all communities have been planted, we apply the "outside" noise to achieve the $d_{\text {out }}$ density among the edges that are between nodes in different communities and between nodes that in the tail of the same community. The full process is detailed in Algorithm 1.

Notice that this model assumes that the "inside" noise is the same in all communities, and the "outside" noise is uniform throughout the graph (though these two types of noise can be very different, and in general, $d_{\text {in }} \gg d_{\text {out }}$ ). We also assume the size and shape of the communities to be uncorrelated.

\section{Time complexity}

Let us analyse the time complexity of Algorithm 1 . For a community $C$, denote by $E_{C}^{p}$ the edges of a "perfect" community (i.e. one with no noise) and denote by $E^{p}$ be the 


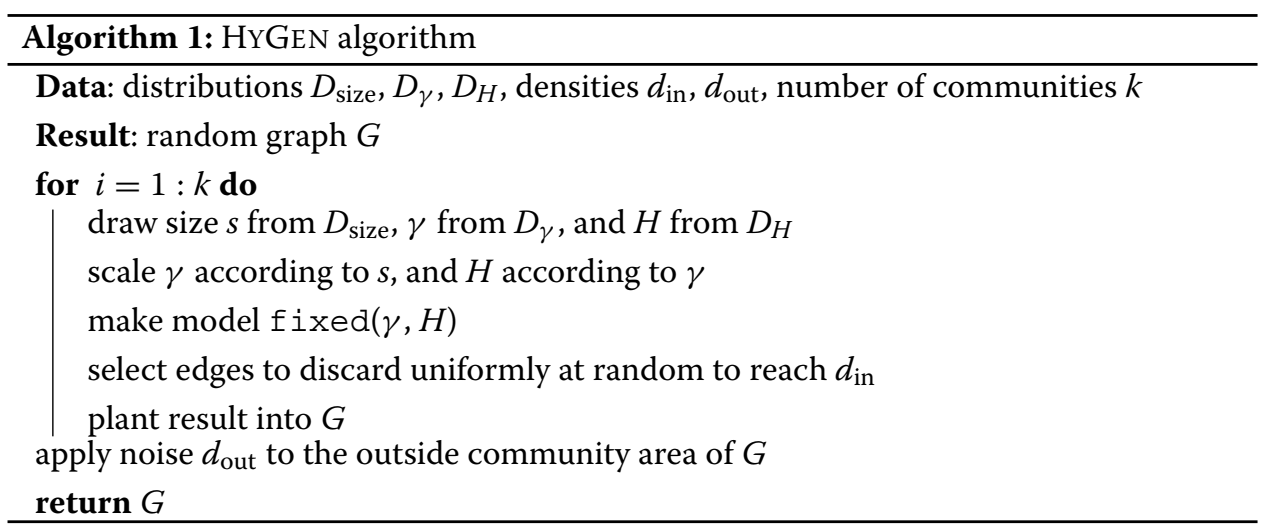

edges in a graph consisting only perfect communities. Let $E^{n}$ be the set of edges noise adds to the graph (inter- and intra-community). Drawing the parameters and adjusting them is a constant-time operation, creating the model takes $O\left(n_{C}\right)$ time, and sampling the edges to discard from the community can be done in time $O\left(\left|E_{C}^{p}\right|\right)$ (Knuth 1981, p. 137). Assuming we have $k$ communities, the total time to generate a graph with no noise is $O\left(k\left(1+n_{c}+\left|E_{C}^{p}\right|\right)\right)=O\left(k+|V|+\left|E^{p}\right|\right)$. To add noise, we need to do sampling without replacement over a population of $O\left(|V|^{2}-\left|E_{p}\right|\right)$ edges, taking essentially linear time. When $\left|E_{p}\right|$ and $\left|E^{n}\right|$ are small compared to $|V|^{2}$ (i.e. the graph is sparse), we can sample with replacement to obtain practically the same result, taking $O\left(\left|E^{n}\right|\right)$ time. In total, the full running time of Algorithm 1 is $O\left(k+|V|+\left|E^{p}\right|+\left|E^{n}\right|\right)$, which is only slightly more than $O(|V|+|E|)$.

\section{HYGEN as a Graphon}

A graphon (Orbanz and Roy 2015) is a measurable function $g:[0,1] \times[0,1] \rightarrow[0,1]$. Graphon $g$ can be seen as a model for random graphs: to sample a graph $G$ from graphon $g$, one first samples $n$ vertices by sampling $n$ points from the unit interval $[0,1]$ uniformly at random (that is, $V \subset[0,1])$. One then samples the edges so that $G$ has edge $(u, v)$ with probability $g(u, v)$. For undirected $G, g$ has to be symmetric, that is, $g(u, v)=g(v, u)$, and the undirected edge between $u$ and $v$ is sampled as the directed edge $(u, v)$.

The HYGEN model for one community is easy to express as a graphon. The graphon is parameterised with four parameters, $p_{R}, \theta_{R}, d_{\text {in }}$, and $d_{\text {out }}$. The parameters $p_{R}$ and $\theta_{R}$ define the hyperbola as with the standard model: given two nodes $u, v \in[0,1]$, the edge between them is part of the community (i.e. $u$ and $v$ are in the intra-community area of the community) if

$$
\left(u+p_{R}\right)\left(v+p_{R}\right) \leq \theta_{R} .
$$

Parameters $d_{\text {in }}$ and $d_{\text {out }}$ are as with the standard model, that is, they define the (expected) inside density and (expected) outside density. The graphon for the HYGEN model for one community is

$$
h= \begin{cases}d_{\text {in }} & \text { if }\left(u+p_{R}\right)\left(v+p_{R}\right) \leq \theta_{R} \\ d_{\text {out }} & \text { otherwise }\end{cases}
$$

Notice that the parameters $p_{R}$ and $\theta_{R}$ are not the same $p$ and $\theta$ as in the hyperbolic model. To understand the difference, it is easier to study the graphon equivalent of the 
fixed model. In the standard fixed model, the parameter $\gamma$ indicates the size of the core; in the graphon version, it indicates the relative size of the core, that is, which fraction of the nodes belongs to the core. Similarly, the $H$ parameter in the graphon model corresponds to the relative thickness of the tail. We denote these parameters as $\gamma_{R}$ and $H_{R}$, respectively. They can be derived from the hyperbola analogously to the standard case (see Metzler et al. (2016)): we can set $\gamma_{R}$ as the value $u^{\prime}$ where $\left(u^{\prime}+p_{R}\right)\left(u^{\prime}+p_{R}\right)=\theta_{R}$ and $H_{R}$ as the value where $\left(u^{\prime}+p_{R}\right)\left(1+p_{R}\right)=\theta_{R}$. This gives

$$
\begin{aligned}
& \gamma_{R}=-p_{R} \pm \sqrt{\theta_{R}} \\
& H_{R}=-\frac{p_{R}^{2}+p_{R}-\theta_{R}}{p_{R}+1}
\end{aligned}
$$

As we require that $\gamma_{R}, H_{R} \in[0,1]$, if $p_{R} \geq 0$, say, then (7) implies that it must be that $\theta_{R} \geq p_{R}\left(p_{R}+1\right)$ and then (6) takes from $\gamma_{R}=-p_{R}+\sqrt{\theta_{R}}$. Similar conditions can be easily derived for $p_{R}<0$ (see Metzler et al. (2016) for the conditions in the standard model).

Vice versa, we can also solve $p_{R}$ and $\theta_{R}$ from $\gamma_{R}$ and $H_{R}$ (cf. Eqs. (2) and (3)):

$$
\begin{array}{lr}
p_{R}=\frac{\gamma_{R}^{2}-H_{R}}{-2 \gamma_{R}+H_{R}+1} & \text { if } \gamma_{R} \neq 1 \text { and } H_{R} \neq 1 \\
\theta_{R}=\frac{\left(\gamma_{R}-1\right)^{2}\left(\gamma_{R}-H_{R}\right)^{2}}{\left(-2 \gamma_{R}+H_{R}+1\right)^{2}} & \text { if } \gamma_{R} \neq 1 \text { and } H_{R} \neq 1 .
\end{array}
$$

When $\gamma_{R}=H_{R}=1$, we can set, for instance, $p_{R}=0$ and $\theta_{R}=1$.

Using graphons in the HYGEN algorithm is straight forward: we generate a graphon for each community based on its parameters and replace the community generation inside the for-loop of Algorithm 1 with sampling the community from its graphon, as explained above. In addition, the graphon model is particularly useful for modelling time-evolving communities. Unlike the standard HYGEN algorithm, the graphon model makes it easy to adjust the size of the community while retaining some vertices: this can be done by simply randomly discarding some of the previously-selected nodes and potentially sampling new ones. Generating a full time-evolving graph would then amount to running Algorithm 1 for every time step, but instead of generating the communities from the scratch, we adjust their sizes based on the graphon model.

Unlike the standard HYGEN model, the graphon model has somewhat more randomness. The parameter $\gamma_{R}$, for example, does not define the actual size of the core, but the expected size of the core. The actual size of the core is a binomially distributed random variable with parameters $\gamma_{R}$ and $s$, where $s$ is the size of the community. Similarly, the density parameters $d_{\text {in }}$ and $d_{\text {out }}$ are only expectations.

\section{HYGEN graphs with specific community structures}

In the above, we described HYGEN model using randomly sampled shape parameters. Normally, we would fit the hyperparameters of the distributions to some real-world data sets to obtain a realistic model for the random graphs, but in some cases, we might want to obtain specific community structures.

One specific task is to generate random graphs with power law connectivity within the communities (similar to the HyCom model of Araujo et al. (2014)). That is easy to do via 
the third model presented by Metzler et al. (2016), the mixture model. That model has again two parameters, $x \in[-1,1]$ and $\Sigma \in \mathbb{R}$, and the function $f$ defining the shape is

$$
f_{\text {mixture }}(i, j, x, \Sigma)=(1-|x|)(i \cdot j)+x(i+j) \leq \Sigma .
$$

The mixture model is, again, equivalent to the fixed and hyperbolic models (Metzler et al. 2016). By setting $x=0$, it becomes clear that the model reduces to a power law; the exponent of the power law is absorbed by the parameter $\Sigma$, as setting $\Sigma^{\prime}=\Sigma^{1 /-\alpha}$ (assuming $\Sigma>0$ ) yields to

$$
(i j)^{-\alpha} \leq \Sigma^{\prime}
$$

Hence we can sample power law communities by just sampling one parameter, $\Sigma \in$ $(0, \infty)$.

A clique-like community is even more restricted than power law community, as we can set $\gamma=H=n_{C}$ to obtain a clique. With such fixed settings, our model reduces to a variation of SBM.

\section{Theoretical results on HYGEN random graphs}

The HYGEN model is designed to preserve important graph properties. In addition to the hyperbolic community structure, HYGEN also preserves the degree distribution and the clustering coefficient of the graph. These two properties measure the connectivity of networks (Aggarwal and Wang 2010), and are often studied with social networks. In this section, we present theoretical analysis of the HYGEN random graphs, proving that they preserve the degree distribution and clustering coefficients (up to noise).

In the last part of this section, we discuss how the hyperbolic community structure determines the degree correlation. This measure is studied to assess to what extent nodes of similar degree connect to each other.

\section{Degree distribution}

The degree distribution is perhaps the most important global property of the graph and has been one of the main topics in many seminal papers (e.g. Watts and Strogatz (1998); Faloutsos et al. (1999); Albert and Barabási (2002)), and preserving the distribution (at least approximately) is one of the standard aims of random graph generators.

As the HYGEN graphs have disjoint communities, we will first analyse a single community. For the sake of simplicity, we will study the complementary cumulative degree distribution $\bar{F}:\{0, \ldots,|V|\} \rightarrow[0,1]$, where $\bar{F}(k)$ is the fraction of vertices with degree at least $k$. Clearly, the cumulative degree distribution $F$ is $F(k)=1-\bar{F}(k)$. For a single community with no noise we have the following lemma.

Lemma 1 Let $C=\left(V_{C}, E_{C}\right)$ be a community that follows the hyperbol ic $(p, \theta)$ model perfectly. The complementary cumulative degree distribution $\bar{F}_{C}$ of $C$ is determined by the parameters $p$ and $\theta$.

Proof According to the model, an edge $(i, j)$ is in $E_{C}$ if and only if $(i+p)(j+p) \leq \theta$ (recall that the model assumes that the vertices are sorted by degree). Re-writing, we obtain that $(i, j) \in E_{C}$ if

$$
j \leq \theta /(i+p)-p .
$$


That is, vertex $i$ has degree at least $j$ if (11) holds, and conversely,

$$
\bar{F}_{C}(j)=\max \{i \in \mathbb{N}: j \leq \theta /(i+p)-p\} /\left|V_{C}\right| .
$$

Lemma 1 uses the hyperbolic model for the simplicity of the proof. Thanks to the the equivalence relations (2) and (3) (see also Metzler et al. (2016)), we can also state the lemma using the potentially more intuitive fixed model and its parameters:

Corollary 1 The complementary cumulative degree distribution of $C, \bar{F}_{C}$ is determined by the parameters $H$ and $\gamma$.

Lemma 1 shows that the degree distribution of a single community is determined by the model; to extend that to the full graph, it is enough to notice that as the communities are disjoint, the total degree distribution is the sum of the individual communities' degree distributions.

Lemma 2 Given a graph $G=(V, E)$ that is a product of the HYGEN model with no noise, its complementary cumulative degree distribution $\bar{F}$ is completely determined by the parameters of its communities.

Proof Let $G$ consist of $k$ communities $C_{1}=\left(V_{C_{1}}, E_{C_{1}}\right), \ldots, C_{k}=\left(V_{C_{k}}, E_{C_{k}}\right)$ and denote by $n_{C_{i}}=\left|V_{C_{i}}\right|$ the number of nodes in community $i$. As the communities are disjoint, and there are no vertices outside the communities,

$$
n=|V|=\sum_{i=1}^{k} n_{C_{i}} .
$$

That there is no noise means that (1) communities are perfect in the sense of Lemma 1, and (2) there are no inter-community edges. Hence, by Lemma $1, \bar{F}_{C_{i}}$ is defined by the parameters of community $C_{i}$, and

$$
\bar{F}(j)=\frac{1}{n} \sum_{i=1}^{k} n_{C_{i}} \bar{F}_{C_{i}}(j),
$$

that is, the number of nodes in $G$ with degree at most $j$ is the sum of the numbers of nodes with degrees at most $j$ over all the communities.

Lemmata 1 and 2 cover the case where there is no noise. Such an assumption is usually too strict for real-world graphs, and would yield to bad modelling of real-world phenomena. When we add the noise, the model parameters (e.g. $p$ and $\theta$ ) will not be enough to define the overall edge distribution. We can show, however, that the noise has most effect to the tails of the degree distribution.

Lemma 3 Let $G$ be a HYGEN graph with no noise and $G^{\prime}$ the same graph with a fraction of $q \in[0,1]$ noise applied, that is, $d_{\text {out }}=q$ and $d_{\text {in }}=1-q$ in $G^{\prime}$. The expected degree of vertex $v$ in $G^{\prime}, \mathbb{E}_{G^{\prime}}[d(v)]$, is

$$
\mathbb{E}_{G^{\prime}}[d(v)]=d(v)+q(n-2 d(v))
$$

where $d(v)$ is the degree of $v$ in $G$ and $n$ is the number of vertices in $G$. 
Proof A fraction of $q$ edges connected to $v$ will be removed due to the noise, and a fraction of $q$ edges not connected to $v$ will be added. Hence,

$$
\mathbb{E}_{G^{\prime}}[d(v)]=d(v)-q d(v)+q(n-d(v)),
$$

which simplifies to (13).

Equation 13 already hints that if $d(v)$ is large or small, the expected degree can have bigger relative changes. Let $\alpha(v)=d(v) / n$, that is $\alpha(v)$ is the relative degree of $v$. Then we can write (13) as

$$
\mathbb{E}_{G^{\prime}}[\alpha(v)]=\mathbb{E}_{G^{\prime}}[d(v)] / n=\alpha(i)+q(1-2 \alpha(i)),
$$

showing that the noise has the most effect on nodes with $\alpha(v) \approx 0$ or $\alpha(v) \approx 1$; on the contrary, if $\alpha(v)=1 / 2$, the presence of noise will have no effect on the expected degree.

The above result means that communities that have many vertices with either very few or very many neighbors are most affected by the noise. An extreme example of such a community would be a star, and similarly, communities with steep power-law curves in degree distribution would also see significant changes from small amounts of noise.

\section{Clustering coefficient}

In addition to the degree distribution, the clustering coefficient is often used to measure the connectivity of the graphs, and high clustering coefficients are associated with "smallworld graphs" (Watts and Strogatz 1998).

There exists two different versions of the clustering coefficient, the global clustering coefficient and the local clustering coefficient (Aggarwal and Wang 2010).

Definition 1 Given a graph $G$, its global clustering coefficient $c c(G)$ is defined as

$$
c c(G)=\frac{\text { number of closed triplets in } G}{\text { number of all triplets in } G} \text {. }
$$

Definition 2 Given an undirected graph $G=(V, E)$ and its vertex $v \in V$, the local clustering coefficient $c_{\nu}(G)$ of $v$ in $G$ is defined as

$$
c c_{v}(G)=\frac{2|\{(u, w): u, w \in \Gamma(v),\{u, w\} \in E\}|}{d(v)(d(v)-1)},
$$

where $\Gamma(v)$ is the neighbourhood of $v$.

We can again show that, up to the effects of the noise, the clustering coefficients - both local and global - are fully determined by the model parameters. We start by analysing the local clustering coefficient in a single community, as that is the most straight forward to do.

Lemma 4 Let $C=\left(V_{C}, E_{C}\right)$ be a community that follows the hyperbolic $(p, \theta)$ without any noise and let $i$ be an arbitrary vertex of $C$. The local clustering coefficient $c c_{i}(C)$ of $i$ in $C$ is determined entirely by $p$ and $\theta$.

Proof Equation 16 has three terms that determine the clustering coefficient: neighbourhood $\Gamma(i)$, set of edges $E_{C}$, and degree $d(i)$. Given that $C$ follows hyperbolic $(p, \theta)$, we can use (11) to express the neighbourhood of $v$ as 


$$
\Gamma(i)=\left\{h \in V_{C}: h \leq \theta /(i+p)-p\right\}
$$

and the set of edges $E_{C}$ as

$$
E_{C}=\left\{i, j \in V_{C}: j \leq \theta /(i+p)-p\right\},
$$

showing that they both depend only on $p$ and $\theta$. That the degree of vertex $i, d(i)$, depends only on $p$ and $\theta$ follows from Lemma 1.

To analyse the global clustering coefficient $c c$, we will first make some definitions. Define the indicator function $\chi(i, j)$ as

$$
\chi(i, j)= \begin{cases}1 & j \leq \theta /(i+p)-p \\ 0 & \text { otherwise } .\end{cases}
$$

This function indicates for every pair of vertices $i$ and $j$ if there would be an edge between them in a community following the hyperbolic $(p, \theta)$ model with no noise.

The number of closed triplets in a community following noise-free hyperbol ic $(p, \theta)$ model can be counted by testing whether all of the edges between the vertices exist. Define $T^{\mathrm{cl}}: \mathbb{N}^{3} \rightarrow\{0,1\}$ as

$$
T^{\mathrm{cl}}(i, j, h)=\chi(i, j) \cdot \chi(i, h) \cdot \chi(j, h) .
$$

An open triplet (wedge) is a set of three vertices connected by exactly two edges. Define $T^{\mathrm{o}}: \mathbb{N}^{3} \rightarrow\{0,1\}$ as

$$
T^{\mathrm{o}}(i, j, h)=\chi(i, j) \cdot \chi(i, h) \cdot(1-\chi(j, h))
$$

to test whether $(i, j, h)$ is an open triplet centred at $i$.

Lemma 5 Let $C$ be as in Lemma 4. The global clustering coefficient cc $(C)$ of $C$ is entirely determined by $p$ and $\theta$.

Proof As the value of $\chi i, j$ is defined by $p$ and $\theta$ (with fixed $i$ and $j$ ), so are the values of $T^{\mathrm{cl}}$ and $T^{\mathrm{o}}$. We can write (15) with them as

$$
c c(C)=\frac{\sum_{i, j, h \in V_{C}} T^{\mathrm{cl}}(i, j, h)}{\sum_{i, j, h \in V_{C}}\left(T^{\mathrm{cl}}(i, j, h)+T^{\mathrm{o}}(i, j, h)\right)},
$$

where we always assume that $i, j$, and $h$ are disjoint.

The above Lemmata 4 and 5 can be extended to a full graph following noise-free HYGEN model.

Lemma 6 Let $G=(V, E)$ be a graph that follows the HYGEN model with no noise. The global clustering coefficient $c c(G)$ and the local clustering coefficient $c c_{v}(G)$ for any $v \in V$ are determined through the parameters of the hyperbolic communities of $G$.

Proof As there are no inter-community edges, the neighbourhood of any $v \in V$ is entirely contained in the community where $v$ is, and Lemma 4 applies directly. The function $\chi$, testing whether there is an edge between vertices $i$ and $j$, also needs to take the communities into account. It can be re-defined as 
Table 1 List of symbols

\begin{tabular}{ll}
\hline Symbol & Meaning \\
\hline$p$ & Center of the hyperbola in the hyperbolic model \\
$p_{R}$ & Center of the hyperbola in the graphon hyperbolic model \\
$\theta$ & Boundary of the hyperbola in the hyperbolic model \\
$\theta_{R}$ & Boundary of the hyperbola in the graphon hyperbolic model \\
$\gamma$ & Size of the core in the $\mathrm{f}$ ixed model \\
$\mathrm{H}$ & Thickness of the tail in the $\mathrm{f}$ ixed model \\
$\gamma_{R}$ & Relative size of the core in the graphon $\mathrm{f}$ ixed model \\
$H_{R}$ & Relative thickness of the tail in the graphon $\mathrm{f}$ ixed model \\
$d_{\text {in }}$ & Density of edges inside the community \\
$d_{\text {out }}$ & Density of the edges outside the community \\
$\mathbb{N}$ & Set of natural numbers $\{0,1,2, \ldots\}$ \\
\hline
\end{tabular}

$$
\chi(u, v)= \begin{cases}1 & \text { if } u, v \in C \text { for some community } C \text { and } \pi_{C}(u) \leq \theta_{C} /\left(\pi_{C}(v)+p_{C}\right)-p_{C} \\ 0 & \text { otherwise }\end{cases}
$$

where $p_{C}$ and $\theta_{C}$ are the parameters of the community $C$ and $\pi_{C}$ is the permutation associated with it. With this definition of $\chi$, the functions $T^{\mathrm{cl}}$ and $T^{\mathrm{o}}$ will also work throughout the full graph, concluding the proof.

Lemma 6 also shows that the average local clustering coefficient is determined by the community parameters.

Corollary 2 Let $G$ be as above. The average local clustering coefficient $\overline{c c}(G)=$ $\frac{1}{|V|} \sum_{v \in V} c c_{v}(G)$ is entirely determined by the community parameters.

It is not trivial to analyse the effects of noise to the clustering coefficient. Triangles or wedges from the inside-community area disappear, and new ones get introduced involving the outside-community area. Given the overall density of a graph, the expected number of triangles or wedges is derivable, but integrating the specific intra-community structure into this expectation remains an open problem.

\section{Degree correlation}

The degree correlation measures whether the number of links between nodes with high degree and nodes with low degree is systematically different from what is expected in a random network. A network is called assortative if nodes of similar degree tend to link to each other, and dissassortative if the network exhibits a preference for links between nodes of dissimilar degree. Both trends of correlation may occur in real world networks. While social networks tend to be assortative, other kinds of networks, including those of question-answering portals, are typically observed to be disassortative (Aggarwal and Wang 2010).

Positive degree correlation in social networks occurs since the degree-ordered adjacency matrix typically shows a banded structure, meaning that links between nodes of similar degree occur more likely than other links. The modular character of such networks with multiple, potentially distinct, communities helps assortativity. Interestingly, for the building blocks of such modular networks, the individual communities, we observe an opposite trend of degree correlation. 
Using results of prior work, we may claim that hyperbolic communities are usually disassortative: Jonhson et al. (2013) study the relation between nestedness of networks and disassortivity. They show that with high probability, disassortative networks are nested and vice versa. Let $G=(V, E)$ be an undirected graph. We say $G$ is nested if we can order the vertices $v \in V$ in a sequence $\left(v_{1}, v_{2}, \ldots, v_{n}\right)$ such that $N\left(v_{i+1}\right) \subseteq N\left(v_{i}\right)$ for all $i=1, \ldots, n-1$. As discussed by Karaev et al. (2018), hyperbolic communities are a special case of nested matrices. Notice that also communities whose adjacency is described by a power law function are therefore disassortative since the power law is special case of the hyperbolic model.

\section{Experimental evaluation}

In this section we present the results of our empirical evaluation. We will first explain the data sets, then study how the parameters are distributed in real-world graphs, and then we study how the other random graph generators work at modelling the kind of communities observed in real-world graphs. We will then study the quality of the graphs constructed using the HYGEN model: first, we will study how well the generated graphs fit to the model and then we will study how random the generated graphs are. Finally, we will study how good the graphon model is at modelling time-evolving communities.

We base our evaluation on the HYGEN parameters. Up to noise, clustering coefficient as well as degree distribution are derivable from the HYGEN parameters, as we have established in the previous section. We do not test inter-community connections such as path length, modularity, or degree correlation, since these would measure only added noise, or in case of the degree correlation of individual communities, be already predetermined through the structures we allow.

\section{Datasets}

We use two different collections of real-world data sets. The first collection, called SNAP, consists of four networks with community information from the Stanford Large Network Dataset collection (Leskovec and Krevl 2014): Amazon, DBLP, Friendster, and YouTube. These are real-world social networks with ground-truth communities labeled in the data. The properties of the graphs are summarised in Table 2.

Our second collection of real-world datasets is called SE and it contains four time-evolving communities from the https://stackexchange.com family of online question-answer sites (Q\&A sites) (Stack Exchange 2016). The communities are https://gaming.stackexchange.com, https://gardening.stackexchange.com, https://tex. stackexchange.com and https://unix.stackexchange.com. The data are from Metzler et al. (2019). Some basic properties of these communities are listed in Table 3. The data sets

Table 2 Summary of the networks in the SNAP collection

\begin{tabular}{lllll}
\hline Network & Nodes & Edges & \multicolumn{2}{c}{ Communities } \\
\cline { 4 - 5 } & & & All & $100-1000$ \\
\hline Amazon & 334863 & 925872 & 75149 & 1380 \\
DBLP & 317080 & 1049866 & 13477 & 805 \\
Friendster & 65608366 & 1806067135 & 957154 & 19763 \\
YouTube & 1134890 & 2987624 & 8385 & 129 \\
\hline
\end{tabular}


Table 3 Basic properties of four SE communities

\begin{tabular}{|c|c|c|c|c|}
\hline Community & Start date & Largest size & Average $\gamma_{R}$ & Average $H_{R}$ \\
\hline Gaming & 2009-08-01 & 3818 & 0.106 & 0.004 \\
\hline Gardening & 2010-07-01 & 446 & 0.124 & 0.009 \\
\hline Tex & 2008-08-01 & 3342 & 0.074 & 0.009 \\
\hline Unix & 2008-08-01 & 5450 & 0.073 & 0.001 \\
\hline
\end{tabular}

The average $\gamma_{R}$ and average $H_{R}$ are computed using a weighted average with the community size at each month being the weight. The parameters $\gamma$ and $H$ for these communities are by Metzler et al. (2019)

contain a snapshot of the community at the begin of each month from the start date until November 2016.

\section{Distributions for the parameters}

Above, we suggested distributions to use for the distributions of the shape parameters $\gamma$ and $H$ and the size $n_{C}$. Here we detail how these suggestions were obtained.

For these experiments, we use the SNAP collection of real-world data sets. We sample 500 communities of size between 100 and 1000 nodes $^{2}$ and compute the hyperbolic models for each community to obtain empirical distributions for $\gamma, H$, and the (truncated) community size $n_{C}$.

For each of the empirical distributions for $\gamma, H$, and $n_{C}$, we fit different distributions (namely, generalized extreme value distribution, inverse Gaussian distribution, Birnbaum-Saunders distribution, exponential distribution, log-normal distribution, loglogistic distribution, gamma distribution, Rayleigh distribution, Weibull distribution, Nakagami distribution, Rician distribution, normal distribution, logistic distribution, extreme value distribution, and $t$-location-scale distribution). Not every distribution is applicable for each of the parameters. While the observed $\gamma$ s look normally distributed, $H$ and the community size show an exponential behaviour.

In order to validate our subjective observations, we tested how well the different distributions fit using the negative log-likelihood (LL). Alternative reasonable measures for evaluation would be Akaike or Bayesian information criterion. We observe highly similar results with either of the measures and therefore only present the evaluation with respect to LL. To show the results in a concise manner, we present here only comparisons of our chosen distributions against all other options. Namely, for every distribution $D$ that is not the one we chose to model parameter $p$, we compute our test statistics $T_{p}(D)=L L_{p}(D)-L L_{p}\left(D^{*}\right)$, where $L L_{p}(D)$ is the negative log-likelihood of modelling parameter $p$ using distribution $D$ and $L L_{p}\left(D^{*}\right)$ is that for the selected distribution $D^{*}$. The larger values $T_{p}(D)$ obtains, the better the selected distribution performs compared to distribution $D, T_{p}(D)=0$ indicates that $D$ and $D^{*}$ perform equally well, and negative values indicate that $D$ performs better than the chosen distribution $D^{*}$.

Based on our experiments, we propose to model $\gamma$ (relative to the community size) using the normal distribution, $H$ (relative to $\gamma$ ) using the exponential distribution, and the community size $n_{C}$ using the generalized extreme value distribution. Our comparison of these distributions against others in the SNAP datasets are presented in Fig. 2.

As can be seen in Fig. 2a, the normal distribution is constantly at least as good as the other distributions (i.e. there are no negative values), implying that the use of normal

\footnotetext{
${ }^{2}$ Notice that the YouTube network has only 129 communities within that size range; hence we use them all.
} 


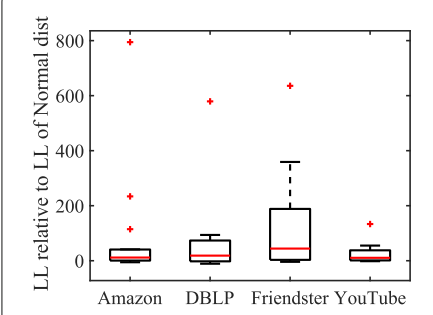

(a)

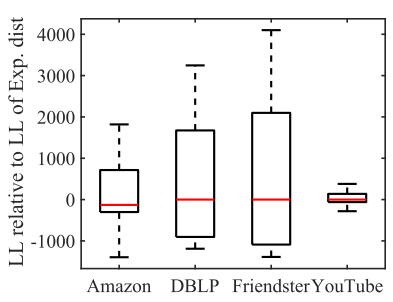

(b)

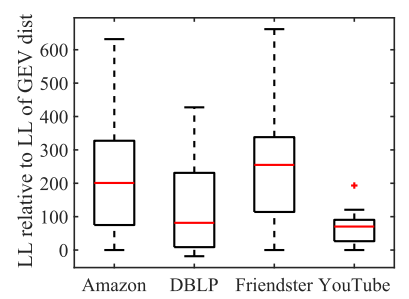

(c)

Fig. 2 Relative log-likelihoods (LL) of the hypothesised distributions compared to the other distributions. For each dataset, the boxplot indicates how well each parameter follows the hypothesised distribution compared to other potential distributions. Positive values indicate that the preferred distribution performs better than the others and zero indicates even performance. $\mathbf{a} \gamma \mathbf{b} \mathrm{H}$ c size

distribution is a valid choice. The situation when modelling the distribution of the $H$ parameter (w.r.t. $\gamma$ ) is more complicated. Our experiments showed the exponential distribution to have the best fit, but as can be seen in Fig. 2b, in some cases other distributions would be better. More experiments would be needed to give a conclusive answer to the question which distribution explains observed $H$ s best.

For modelling the distribution of the community size (Fig. 2c), the GEV distribution is always the best, and shows the strongest performance against the other distributions. Please note, however, that in this test we model the distribution of the sizes of the communities in a single network at one point of time; when studying the sizes of a single community over time, very different distributions might be needed (cf. Fig. 8).

\section{Limits of the current graph generators}

While many of the existing random graph generators cannot be used to model community structures in networks at all, there are a number of related approaches that allow for generated graphs with community structure. To the best of our knowledge however, there exists no approach of preserving the intra-community connection patterns in the modeling process.

In this section, we detail why SBM, LFR, and R-MAT do not provide solutions to the modeling task we aim to solve. For this purpose, we compare the outcome of these generators when fitted to idealized hyperbolic communities. One may argue that such communities are not realistic and therefore not a fair basis of comparison for these generators that are designed to model real-world graphs, which are usually sparse and exhibit a certain level of noise. However, the purpose of this experiment is to see to what extent the pure hyperbolic structure can be captured at all by the different generators.

Experimental Setup. We generate single hyperbolic communities with the extremes of shapes that can be represented: a star, a near-clique, a triangular, and a stereotypical coretail pattern (see Fig. 3; cf. Fig. 1b). Each of these communities consists of 100 nodes. For each of the graph generators, SBM, LFR, and R-MAT, we learn the respective parameters with these communities as input and generate new graphs according to those parameters. After that, we fit the best hyperbolic model on the newly generated graphs, measure the fitting quality in terms of log-likelihood (LL), and compare how well the obtained model matches the original input. We repeat this experiment 50 times to counteract effects of 


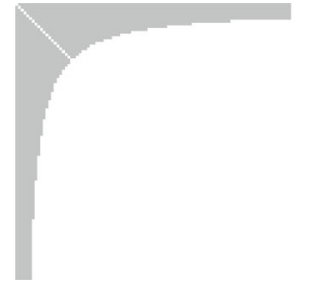

(a)

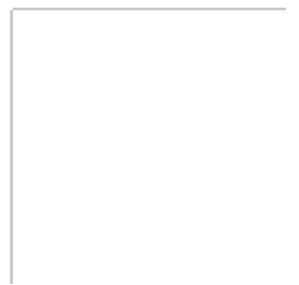

(b)

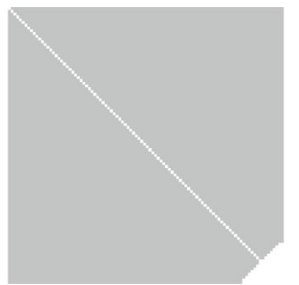

(c)

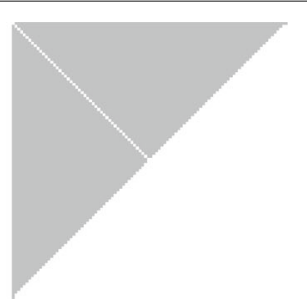

(d)

Fig. 3 Adjacency matrices of idealised hyperbolic communities. The different extremes of hyperbolic structures, range from star-like to near-clique. a 20\%-core b star c near-clique d triangle

randomness. Figure 5 summarizes the resulting hyperbolic models that best fit the communities generated by the respective method. We overlay the 50 obtained models in light grey. Ideally, we would expect to see the exactly the structures of Fig. 3 again.

Notice that the LFR implementation strictly requires multiple communities to be generated. Therefore, we provide graphs containing twice the same community as input (like illustrated in Fig. 6a for the 20\%-core) and use the better matching one in the evaluation of the fit quality.

SBM. The standard SBM is based on the assumption that vertices within a block are stochastically equivalent. The hyperbolic model fulfils this assumption only in the extreme case of a quasi-clique, where the size of the core equals the size of the community. The typical core-tail structure of hyperbolic communities cannot be captured (see Fig. 4a). As the generated graphs after fitting the SBM model are almost uniformly dense, the hyperbolic models fitted on these outputs exhibit a huge degree of variance and often differ a lot from the original input (see Fig. 5a). The high negative LL scores of the fits (see Table 4) indicate that the fitted hyperbolic models are also not particularly good at explaining SBM-generated communities. Regarding the case of a near-clique, it is worth pointing out that a perfect clique would be recovered well by the SBM. The case of the near-clique (Fig. 5a, column 3) is substantially harder: almost all nodes are connected to each other but a few miss some connections. For the SBM however, the primary aim is to match the overall density evenly. Thus the fit is such that many nodes are fully connected and some are connected to almost every other node, which yields into a substantially different looking hyperbolic model.

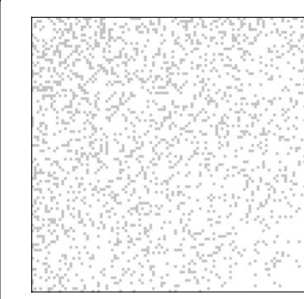

(a)

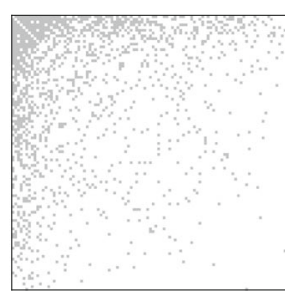

(b)

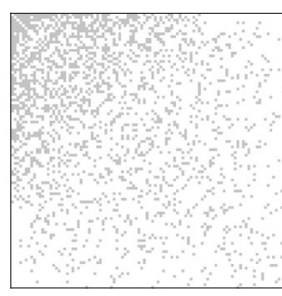

(c)

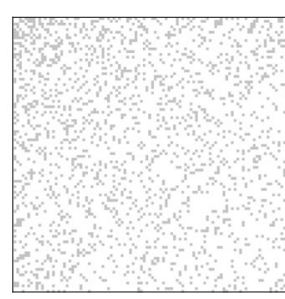

(d)

Fig. 4 How the compared graph generators create a new random community when given the 20\%-core community shown in Fig. 3 as input. The adjacency matrices in each subfigure are degree-ordered. a SBM $\mathbf{b}$ DC-SBM c R-MAT d LFR 


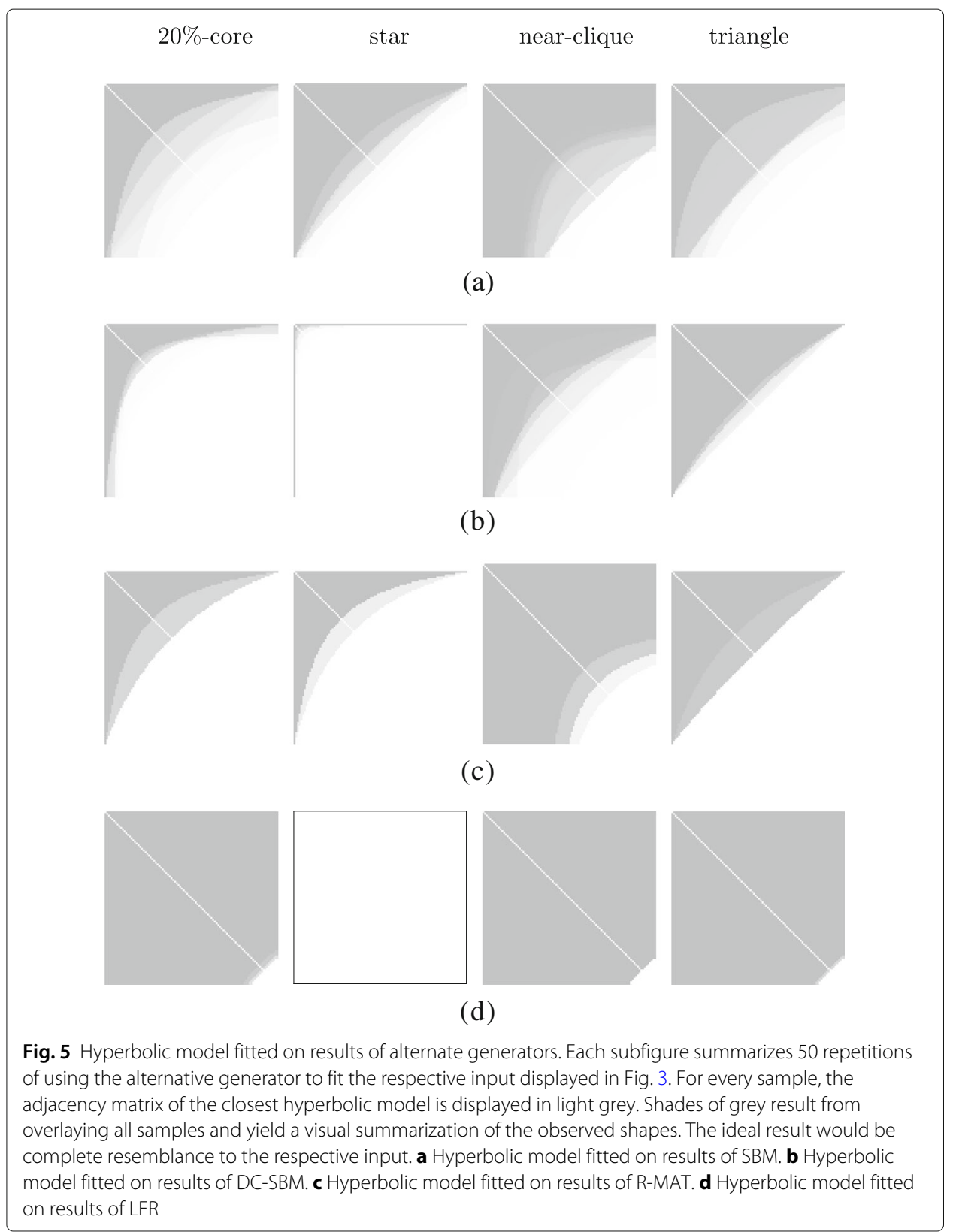

Table 4 Average best log-likelihood for 50 trials of generating hyperbolic communities with SBM, DC-SBM, R-MAT, and LFR, with standard deviations

\begin{tabular}{lllll}
\hline & 20\%-core & star & near-clique & triangle \\
\hline SBM & $-2354(1919)$ & $-451(1406)$ & $-282(1007)$ & $-3393(39)$ \\
DC-SBM & $-1545(1734)$ & $-212(713)$ & $-3238(521)$ & $-2679(768)$ \\
R-MAT & $-2008(38619)$ & $-440(958)$ & $-214(548)$ & $-2727(230133)$ \\
LFR & $-2515(7697)$ & - & $-1299(17346)$ & $-3714(6250)$ \\
\hline
\end{tabular}

Notice that HyGen would achieve the ideal value of 0 in each of the examined cases. 
DC-SBM. DC-SBMs allow for variation of the degree within a community (see Fig. 4b). Fitting back a hyperbolic model on the DC-SBM outcome of modelling a 20\%-core structure, a star, or a triangle are fairly accurate in the sense that the parameters $\gamma$ and $H$ are close to the original (see Fig. 5b). Yet, the hyperbolic models fitted on the DCSBM-generated communities leave some amount of noise to be explained otherwise (see Table 4). The DC-SBM expects a power-law degree distribution within the communities and draws edges from that one to recover the connectivity pattern inside the community. In particular the near-clique case (see Fig. 5b, column 3) seems to be hard to explain by a power law. The hyperbolic model is more general in the sense that it includes power-law distributions as a special case. It also has a substantially different noise model, assuming uniform density for the inside-community area and as well as for the outside.

R-MAT. R-MAT is designed to model the degree distribution of the input data using a recursive procedure. The results we observe for the single artificial communities are comparable to those of the DC-SBM (see Fig. 5c). The recursive construction procedure however introduces particular structures in the data. To construct a graph, R-MAT subdivides the adjacency matrix recursively into quarters of certain density. This makes it hard to capture multiple communities, especially of unequal sizes. An additional experiment reveals that, already if R-MAT is fitted on a graph of two equally sized (hyperbolic) communities with no inter-connections, the resulting model is not capturing the this structure well (see Fig. 6): by its definition, R-MAT models consist of four self-similar blocks. This means, the blocks with community structure are always mirrored to the off-diagonal, introducing many surplus links between the communities.

LFR. The LFR benchmark generates random graphs given power-law distributions for the node degree and the community size. Creating graphs that consist of a single community is not included as a special case in this approach. To still obtain comparable modelling results for the four sample hyperbolic communities (see Fig. 3), we fit LFR on graphs consisting of twice each of those communities. The reported LL scores of fitting a hyperbolic model on the LFR results then refer to the better of the two obtained communities. While for the star pattern, we could not find a set of valid LFR parameters to describe this pattern with the procedure suggested by Lancichinetti et al. (2008), we observe that the remaining

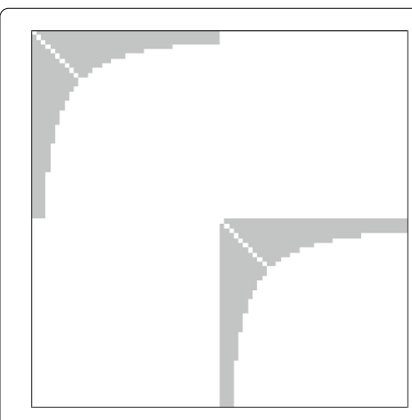

(a)

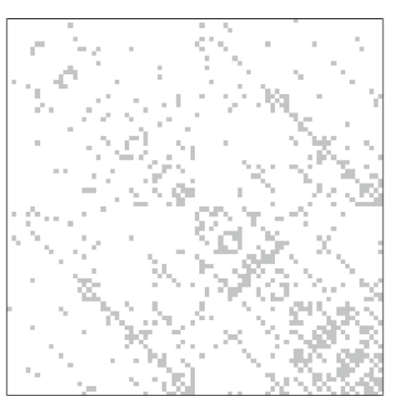

(b)

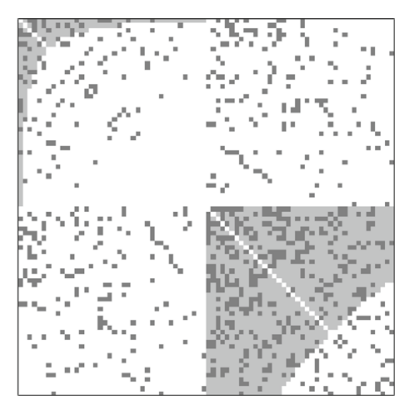

(c)

Fig. 6 How R-MAT reconstructs a graph of two identical hyperbolic communities. To fit the hyperbolic models, we assume that the community sizes remained unchanged during modeling. Fitting implies degree ordering the communities. a input graph b R-MAT result c fitted model 
hyperbolic communities are modeled very similar to each other by LFR, as the best hyperbolic model to explain these communities is the same in each case (see Fig. 5d). A closer look at the LFR-generated graphs reveals that they actually differ: the average degree per community is retained from the original communities, but the hyperbolic structure is lost.

\section{Stability of the graph generation}

We now turn our attention into analysing the HYGEN-generated graphs. In this section we will study how well the generated graphs fit to the real-world graphs; in the next section, we will study how random the generated graphs are.

We used the following procedure to test how well the generated graphs fit to the real-world data they were generated from: First, we fitted the hyperparameters of the parameter distributions to real-world networks from the SNAP collection. Then we used these distributions in HYGEN to sample collections of random graphs. Finally, we computed the best hyperbolic model for each community in the generated graphs using the code of Metzler et al. (2016) and evaluated how accurately the found communities match to the original communities. Our hypothesis is that the found communities have similar distributions of parameters as the original communities, indicating that the generated graphs retain the essence of the community structure of the original graphs.

In order to compare HYGEN, we also generated graphs using LFR and DC-SBM. Based on our experiments in the previous section, we know that they cannot model the hyperbolic structure too well, but it is still possible that they can model all of the structure that is observed in real-world graphs.

In order to fit the hyperbolic model to the generated communities, we need to know what these communities are. Both HYGEN and LFR return this information; the DC-SBM implementation we used (Nepusz 2015), on the other hand, only uses this information internally and does not report it. In order to find the communities from graphs generated by DC-SBM, we will fit the model again to the generated graphs, and record the community structure from the fitted models. We assume that DC-SBM correctly recovers communities when provided graphs generated by it.

The results of this experiment, with respect to $\gamma, H$, and community size, are presented in Fig. 7. It shows four boxplots for each dataset and parameter combination: the first, called empirical, is the distribution observed from the original data, i.e. the distribution we should match, and the other three boxplots show the distributions of the parameters when fitted to the generated graphs.

In order to obtain reliable results, with HYGEN and LFR, we sampled a hundred times as many communities as was in the original data. That is, for the YouTube data, we sampled 12900 communities, and for the other datasets, we sampled 50000 communities. DC-SBM, on the other hand, was so slow to generate the sampled graphs and fit to them that we had to limit it to one graph with 100 communities for each dataset. Even with this limitation, it could not finish the Friendster data set within a week, and hence we exclude DC-SBM from the results regarding the Friendster data.

The results in Fig. 7a show that HYGEN generates a very good match for $\gamma_{R}$ in all datasets. LFR is somewhat accurate, but has much higher standard deviation and often generates too many communities with too large cores. This behaviour is most pronounced in the YouTube dataset. DC-SBM generates communities with even larger cores, and in 


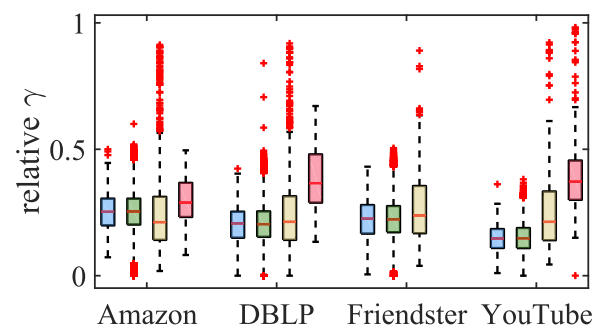

(a)

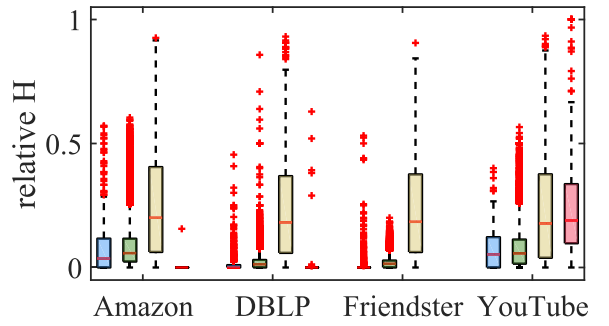

(b)

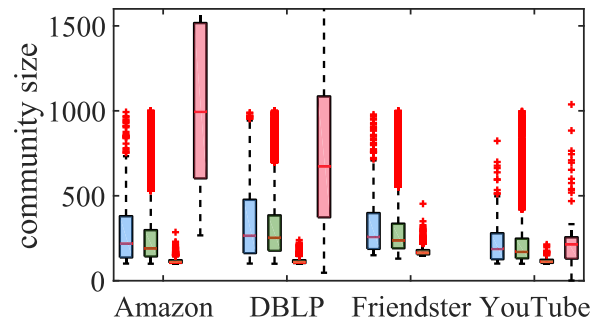

empirical

HYGEN

LFR

DC-SBM

(c)

Fig. 7 Distributions of parameters in generated graphs compared to the empirical distributions observed in the original data. $H$ and $\gamma$ are obtained after fitting hyperbolic models. a $\gamma_{R} \mathbf{b} H_{R} \mathbf{c}$ community size (data for DC-SBM > 1600 not displayed)

case of DBLP and YouTube, the first quartile of $\gamma_{R}$ in DC-SBM generated communities is above the third quartile of the original distribution, indicating a very bad fit.

Figure $7 \mathrm{~b}$ shows the results for the relative $H$. Again, HYGEN produces the most accurate results, although the communities have a slightly thicker tail than in the original data at least in DBLP and Friendster data sets. On the other hand, these data sets have extremely thin tails in their communities. The communities generated by the LFR model have much thicker tails than the real communities or those generated by the HYGEN model. In short, it is obvious that LFR cannot model the kind of thin tail real-world data sets often have. DC-SBM, on one hand, under-estimates the tail thickness for the Amazon data set, and on the other hand, over-estimates it for the YouTube data set.

When we look at the sizes of the generated communities, in Fig. 7c, we can observe that LFR has very small deviation of the community sizes, and they are generally too small, while DC-SBM generates too large communities for Amazon and DBLP data sets, but approximately correctly-sized communities for the YouTube data set. The communities generated by HYGEN have again the best fit in the distribution of the sizes, though it seems to generate slightly less of the larger communities than what is seen in the real data.

Overall, we can conclude that HYGEN provides a reasonably good fit for the original data, and significantly better than what is provided by either LFR or DC-SBM. This again shows that HYGEN is the best method for modelling hyperbolic structure, and that the real-world data actually has communities with structure that cannot be captured by the other models.

\section{Randomness of the generated graphs}

We now turn onto our second evaluation: whether the generated graphs are random enough. In a way, this experiment test the opposite of the previous experiment; it would 
be easy to obtain a very good match to the original graph by simply generating graphs that are identical copies of the original one, but these would be rather useless. Hence, it is important to study also whether the generated graphs have enough randomness.

For this study, we quantify the randomness using the conditional entropy $\mathcal{H}(Y \mid X)$. Intuitively, it measures how much information is needed to describe random variable $Y$ given that we know random variable $X$, that is, how much $X$ "tells about" $Y$. If $Y$ is fully determined by $X, \mathcal{H}(Y \mid X)=0$, and if $Y$ and $X$ are independent, $\mathcal{H}(Y \mid X)=\mathcal{H}(Y)$, the entropy of $Y$ (Cover and Thomas 2006). As $0 \leq \mathcal{H}(Y \mid X) \leq \mathcal{H}(Y)$, we report the relative conditional entropy $\mathcal{H}_{R}(Y \mid X)=\mathcal{H}(Y \mid X) / \mathcal{H}(Y) \in[0,1]$.

We use the relative conditional entropy to compare the adjacency matrices of the different communities (original and generated). As the graphs are undirected, we will only study the upper triangular part of the adjacency matrix. We sort the rows and columns of the adjacency matrix according to the vertex degree so that they are ordered in similar way for all graphs. We then consider the upper triangular part of the adjacency matrix as a binary vector, and identify that as a discrete random variable.

To generate the data to compute $\mathcal{H}_{R}(Y \mid X)$, we generated 100 random graphs using HyGEN. We sampled $\gamma$ and $H$ from fitted distributions, but for the size, we used the original sizes of the communities to ensure that the random variables $X$ and $Y$ are of same length.

The relative conditional entropy $\mathcal{H}_{R}(Y \mid X)$ is computed per community because communities sampled from the original graph do not maintain their context (i.e. which community overlaps with which community), and thus we cannot match the generated communities correctly to the original communities unless we generate the communities one-by-one. In addition, using the fixed sizes of communities introduces determinism not present in the full graph model that could bias the analysis.

Our results are presented in Table 5 . The results indicate that the information we have on the generated graphs given the original data is very small (all relative conditional entropies are larger than 0.96), that is, the generated communities are truly random. Together with the previous experiment, showing that the generation preserves the desired structure, we can conclude that HYGEN can generate random graphs that preserve the desired structure.

\section{Modelling time-evolving communities}

In this section we use the graphon version of the HYGEN model to model time-evolving communities. Communities in social networks, especially in online Q\&A sites, such as https://stackexchange.com, have surprisingly constant relative core size (Metzler et al. 2019). In other words, the relative $\gamma, \gamma_{R}$, stays almost constant over time, indicating that the graphon model should be a good model for such communities. The purpose of this

Table 5 Average relative conditional entropy of generated communities given the original data

\begin{tabular}{lc}
\hline & $\mathcal{H}_{R}(Y \mid X)$ \\
\hline Amazon & 0.996 \\
DBLP & 0.996 \\
Friendster & 0.990 \\
YouTube & 0.963 \\
\hline
\end{tabular}

Standard deviations are within the displayed precision and thus omitted 
experiment is to study whether that is true; in particular, whether the graphon model can generate time-evolving communities that behave similarly to the real ones.

In these experiments, we used the four communities from the SE collection, described in Table 3. Notice that here each dataset is just one community; this is sufficient for this experiment, as multi-community graphs would not change the behaviour of single communities. A graphon was initialised for each community using the relative parameters $\gamma_{R}$ and $H_{R}$ from Table 3 and a new community was sampled for every month in the data. We did not model the size of these communities, but used the real sizes. Similarly, we kept the same number of nodes from the previous month as was kept in the real data. Also, the sampling was done without adding any noise. This way we can concentrate on the shape of the community; the modelling of the size, or the amount of the overlap, over time is an interesting problem for future work (as Fig. 8 shows, there is no common trend in the sizes) and adding the noise will not change the analysis of the shape. As the tail height parameter $H_{R}$ is almost zero for every community (as is the case with essentially all communities like this (Metzler et al. 2019)), we do not report any results on that one.

Figure 8 shows the results for the four SE communities. The blue line shows the true $\gamma_{R}$ for each month (computed by Metzler et al. (2019)). The red line shows the average $\gamma_{R}$ computed from 100 samples from the graphon, and the shaded red area extends two standard deviations above and below the average (though clipping at 0 ).

As we can see in the blue lines, all communities have much higher values of $\gamma_{R}$ at the beginning of their lives but soon the values converge to a lower value and stay rather constant. The biggest reason for the behaviour at the begin seems to be

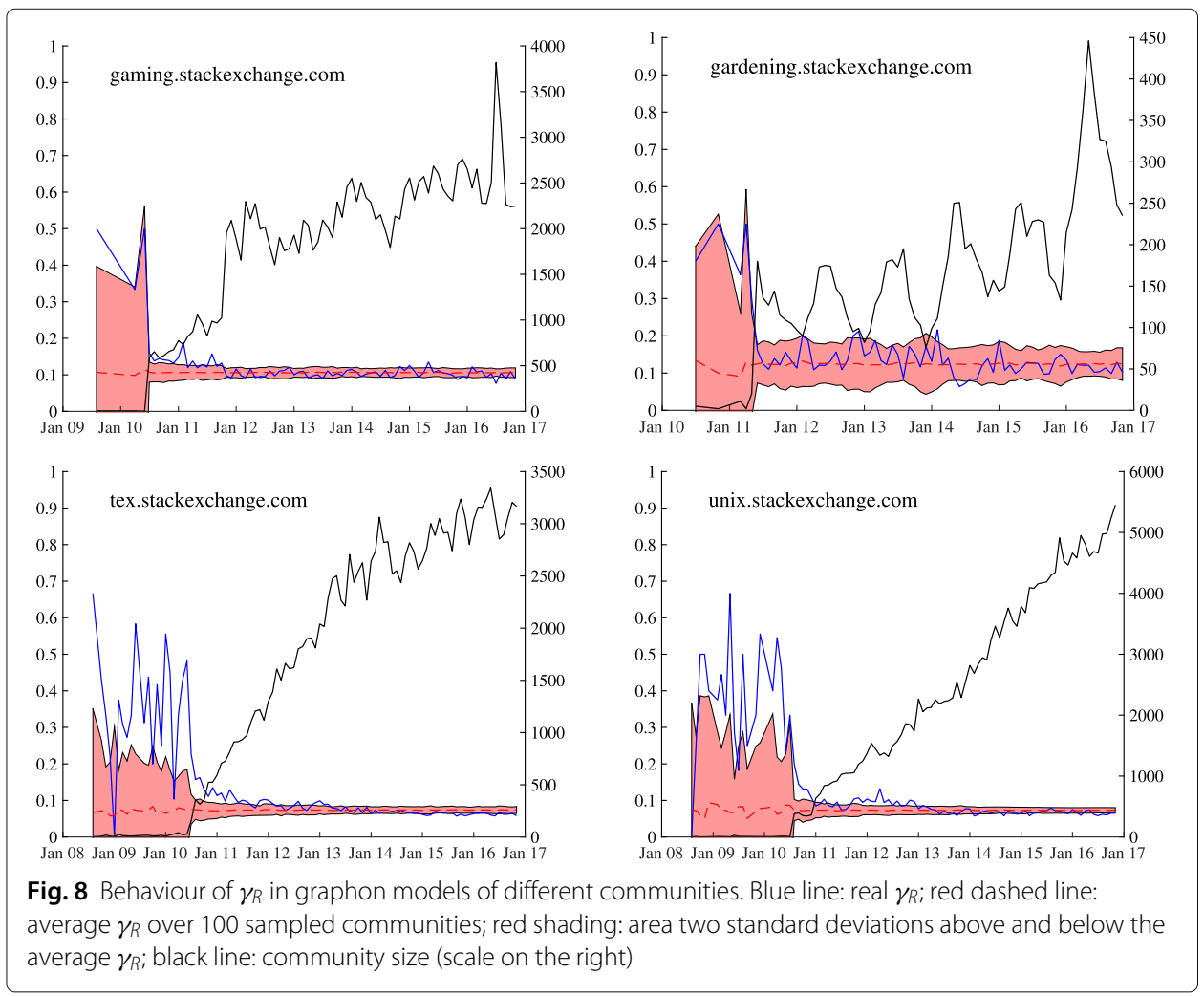


the size of the community (depicted as black line in the figures): initially all communities start small, but as they grow, the relative core size $\gamma_{R}$ stabilises. This can be readily seen, for instance, from the gardening community, that has a cyclic pattern in its size: the value of $\gamma_{R}$ varies the most when the community has the smallest sizes.

The graphon model can capture this variance based on size very well. The larger the sample (i.e. community), the smaller the standard deviation around the expected core size $\gamma_{R}$. Consequently, the samples follow the behaviour of the real graphs very closely, having much higher deviation at the early stages of the communities and stabilizing as the community grows. Notice that the model even follows the individual peaks very well (e.g. in unix community), even if the initial values are usually more than two standard deviations away from the average.

Overall, this experiment shows that the graphon model can be used very effectively to model time-evolving hyperbolic communities, even though there are some important future directions to explore. The current model selects the members that leave the community uniformly at random; potentially more realistic model would depend, for instance, on the length of the node's membership in the community and on its degree. Also, we did not model the community size over time. In order to generate fully synthetic graphs, this is a very important feature, although as can be seen already from Fig. 8, different communities can have so different behaviour that a single distribution is probably never sufficient to model all of them.

\section{Discussion and conclusions}

Being able to generate random graphs with realistic community structure is important for testing community detection algorithms and for understanding how realistic graphs behave. Recent research has shown that real-world communities have much richer structure than the typically-assumed quasi-clique. Our model, HYGEN, is capable of generating random graphs with this richer structure, making them more realistic. The proposed model can also formulated as a graphon; a modelling that is particularly well-suited for modelling time-evolving graphs.

While HYGEN is already an improvement over the state-of-the-art random graph generators, there are still important topics of further development. The first important topic is to incorporate more realistic noise models to HYGEN. At the present, the model assumes uniform noise with different probabilities of eliminating real edges and adding spurious ones. Our experiments, however, indicate that the noise is correlated with the size of the community. Incorporating a size-dependent noise model for removing the true edges is somewhat straight forward, but modelling similar noise for inter-community edges requires future work.

The HYGEN-generated communities have currently too thick tails compared to what we see in the real world. This might be because the distribution we use to model the tail thickness parameter (Exponential) is not concentrated strongly enough, or it might have something to do with the noise model.

Finally, HYGEN produces non-overlapping communities. In principle, this could be solved relatively easily: the HYGEN algorithm (Algorithm 1) could generate partiallyoverlapping communities assuming it knows the amount of overlap. This could be either provided indirectly by specifying number of nodes for the output graph $G$, or by 
specifying the amount of mixture among communities. The real challenge however is not the definition of such a model, but its evaluation. Available test data from real world networks only comes with community information with respect to the nodes. Assuming the hyperbolic model, overlap can either be within the intra-community area, or outside. In both cases, we would observe overlapping nodes, but only in the first case the communities actually overlap. Due to the lack of data to evaluate the realism of generated graphs with overlapping communities, we leave this extension of the model for future work.

HYGEN has its obvious use for testing community detection algorithms. A comprehensive comparison of community detection algorithms, as done by Orman et al. (2013) and Fagnan et al. (2018), is a planned future work. HYGEN can generate realistic graphs equipped with reliable labelling of the communities. Besides this, HYGEN might serve as an anonymization tool to study the structure of social networks without revealing the participants identities.

\title{
Abbreviations
}

DC-SBM: Degree-corrected stochastic block model; GEV: Generalized extreme value; LFR:

Lancichinetti-Fortunato-Radicchi benchmark; LL: Log-likelihood; SBM: Stochastic block model

\section{Acknowledgements}

The authors wish to express their gratitude for Prof. Stephan Günnemann and Dr. Dóra Erdős for helpful discussions during the preparation of this manuscript.

\section{Authors' contributions}

SM designed the HyGen generator, did the theoretical analysis and implementation, and conducted the empirical experiments. PM helped with the design and analysis, designed the graphon version and did its implementation and experimental evaluation. Both authors participated equally on writing of the manuscript. Both authors read and approved the final manuscript.

Funding

Not applicable.

\section{Availability of data and materials}

The datasets used and/or analysed during the current study are available from the corresponding author on reasonable request. All source code used in the current study is implemented in Matlab (tested on Matlab 2018b) and is available from the corresponding author on reasonable request.

\section{Competing interests}

The authors declare that they have no competing interests.

\author{
Author details \\ ${ }^{1}$ Max-Planck-Institute for Informatics, Saarland Informatics Campus, 66123, Saarbrücken, Germany . 2 University of \\ Eastern Finland, P.O. Box 1627, Fl-70211, Kuopio, Finland.
}

Received: 21 March 2019 Accepted: 2 July 2019

Published online: 31 July 2019

\section{References}

Abbe E (2017) Community detection and stochastic block models: recent developments. https://arxiv.org/abs/1703. 10146. Accessed 21 Mar 2019

Aggarwal CC, Wang H (eds) (2010) Managing and Mining Graph Data. Springer, New York

Alba RD, Moore G (1978) Elite social circles. Sociol Methods Res 7(2):167-188

Albert R, Barabási A-L (2002) Statistical mechanics of complex networks. Rev Mod Phys 74:47-97

Araujo M, Günnemann S, Mateos G, Faloutsos C (2014) Beyond blocks: Hyperbolic community detection. In: Calders T, Esposito F, Hüllermeier E, Meo R (eds). Proc. 2014 European Conference on Machine Learning and Principles and Practice of Knowledge Discovery from Databases (ECMLPKDD '14). Springer, Berlin. pp 50-65

Borgatti SP, Everett MG (1999) Models of core/periphery structures. Soc Netw 21:375-395

Chakrabarti D, Zhan Y, Faloutsos C (2004) R-MAT: A Recursive Model for Graph Mining. In: Berry MW, Dayal U, Kamath C, Skillicorn DB (eds). Proc. 4th SIAM International Conference on Data Mining (SDM '04). Society for Industrial and Applied Mathematics, Philadelphia. pp 442-446

Cover TM, Thomas JA (2006) Elements of Information Theory. Wiley, Hoboken

Erdős P, Rényi A (1959) On random graphs I. Publi Math Debrecen 6:290

Fagnan J, Abnar A, Rabbany R, Zaïane OR (2018) Modular Networks for Validating Community Detection Algorithms. http://arxiv.org/abs/1801.01229. Accessed 21 Mar 2019 
Faloutsos M, Faloutsos P, Faloutsos C (1999) On power-law relationships of the Internet topology. In: Chapin L, Sterbenz JPG, Parulkar GM, Turner JS (eds). Proc. ACM SIGCOMM 1999 Conference on Applications, Technologies, Architectures, and Protocols for Computer Communication (SIGCOMM '99). ACM, New York. pp 251-262

Girvan M, Newman MEJ (2002) Community structure in social and biological networks. Proc Nat Acad Sci USA 99(12):7821-7826

Holland P, Laskey K, Leinhardt S (1983) Stochastic blockmodels: First steps. Soc Netw 5(2):109-137

Jonhson S, Domínguez-García V, Muñoz MA (2013) Factors Determining Nestedness in Complex Networks. PLoS ONE 8(9):74025

Karaev S, Metzler S, Miettinen P (2018) Logistic-Tropical Decompositions and Nested Subgraphs. In: 14th International Workshop on Mining and Learning with Graphs, London. http://www.mlgworkshop.org/2018/papers/MLG2018 paper_35.pdf

Karrer B, Newman MEJ (2011) Stochastic blockmodels and community structure in networks. Phys Rev E 83:016107

Knuth DE (1981) The Art of Computer Programming Vol. 2: Seminumerical Algorithms. 2nd. Addison-Wesley, Reading

Krioukov D, Papadopoulos F, Kitsak M, Vahdat A, Boguñá M (2010) Hyperbolic geometry of complex networks. Phys Rev E 82:036106

Lancichinetti A, Fortunato S, Radicchi F (2008) Benchmark graphs for testing community detection algorithms. Phys Rev E 78(4):046110

Laumann EO, Pappi FU (1976) Networks of Collective Action: A Perspective on Community Influence Systems. Academic Press, New York

Leskovec J, Krevl A (2014) SNAP Datasets: Stanford Large Network Dataset Collection. http://snap.stanford.edu/data. Accessed 11 Feb 2016

Leskovec J, Kleinberg J, Faloutsos C (2005) Graphs over time: Densification laws, shrinking diameters and possible explanations. In: Grossman R, Bayardo RJ, Bennett KP (eds). Proc. 11th ACM SIGKDD International Conference on Knowledge Discovery and Data Mining (KDD '05). ACM, New York. pp 177-187

Leskovec J, Chakrabarti D, Kleinberg J, Faloutsos C, Ghahramani Z (2010) Kronecker graphs: An approach to modeling networks. J Mach Learn Res 11:985-1042

Metzler S, Günnemann S, Miettinen P (2016) Hyperbolae are no hyperbole: Modelling communities that are not cliques. In: Bonchi F, Domingo-Ferrer J, Baeza-Yates RA, Zhou Z-H, Wu X (eds). Proc. 16th IEEE International Conference on Data Mining (ICDM '16). IEEE Computer Society, Los Alamitos. pp 330-339

Metzler S, Miettinen P (2019) Random graph generators for hyperbolic community structures. In: Aiello LM, Cherifi C, Cherifi H, Lambiotte R, Lió P, Rocha LM (eds). Proc. 7th International Conference on Complex Networks and Their Applications (COMPLEX NETWORKS '18). Springer, Cham. pp 680-693

Metzler S, Günnemann S, Miettinen P (2019) Stability and dynamics of communities on online question-answer sites. Soc Netw 58:50-58. https://doi.org/10.1016/j.socnet.2018.12.004

Morgan DL, Neal MB, Carder P (1997) The stability of core and peripheral networks over time. Soc Netw 19(1):9-25

Nepusz T (2015) blockmodel: Fitting stochastic blockmodels to empirical networks. https://github.com/ntamas/ blockmodel. Accessed 4 Mar 2019

Orbanz P, Roy DM (2015) Bayesian models of graphs, arrays and other exchangeable random structures. IEEE Trans Patern Anal 37(2):437-461

Orman GK, Labatut V, Cherifi H (2013) Towards realistic artificial benchmark for community detection algorithms evaluation. Int J Web Based Commun 9(3):349

Panzarasa P, Opsahl T, Carley KM (2009) Patterns and dynamics of users' behavior and interaction: Network analysis of an online community. J Am Soc Inf Sci Technol 60(5):911-932

Park H, Kim M-S (2018) EvoGraph: An Effective and Efficient Graph Upscaling Method for Preserving Graph Properties. In: Guo Y, Farooq F (eds). Proc. 24th ACM SIGKDD International Conference on Knowledge Discovery and Data Mining (KDD '18). ACM, New York. pp 2051-2059

Reed PB, Selbee LK (2001) The Civil Core in Disproportionality in Charitable Giving, Volunteering, Civic Participation. Nonprofit Volunt Sect Q 30(4):761-780

Stack Exchange Inc (2016) Stack Exchange Data Dump. https://archive.org/details/stackexchange. Accessed 24 Jan 2017

Watts DJ, Strogatz SH (1998) Collective dynamics of 'small-world' networks. Nature 393(6684):440-442. https://doi.org/10. 1038/30918

Zhang JW, Tay YC (2016) GSCALER: Synthetically Scaling A Given Graph. In: Pitoura E, Maabout S, Koutrika G, Marian A Tanca L, Manolescu I, Stefanidis K (eds). Proc. 19th International Conference on Extending Database Technology (EDBT '16). OpenProceedings.org, Konstanz. pp 53-64

Zhu Y, Yan X, Moore C (2014) Oriented and degree-generated block models: generating and inferring communities with inhomogeneous degree distributions. J Compl Netw 2(1):1-18. https://doi.org/10.1093/comnet/cnt011

\section{Publisher's Note}

Springer Nature remains neutral with regard to jurisdictional claims in published maps and institutional affiliations. 